\title{
Utility of HTLV proviral load quantification in diagnosis of HTLV-1-associated myelopathy requires international standardization
}

\author{
Maria Fernanda Rios Grassi ${ }^{\mathrm{a}, \mathrm{b}, *}$, Viviana Nilla Olavarria ${ }^{\mathrm{b}}$, \\ Ramon de Almeida Kruschewsky ${ }^{\mathrm{b}}$, Yoshihisa Yamano ${ }^{\mathrm{c}}$, Steven Jacobson ${ }^{\mathrm{d}}$, \\ Graham P. Taylor ${ }^{\mathrm{e}}$, Fabiola Martin ${ }^{\mathrm{f}}$, Bernardo Galvão-Castro ${ }^{\mathrm{a}, \mathrm{b}}$ \\ a Advanced Laboratory of Public Health, Gonçalo Moniz Center, Fundação Oswaldo Cruz, Salvador, Bahia, Brazil \\ b Bahiana School of Medicine and Public Health (EBMSP), Salvador, Bahia, Brazil \\ c Department of Rare Diseases Research, Institute of Medical Science, St. Marianna University School of Medicine, Kanagawa, Japan \\ d Viral Immunology Section, NINDS/NIH, Bethesda, MD, United States \\ e Section of Infectious Diseases, Faculty of Medicine, Imperial College London, London, United Kingdom \\ f Centre for Immunology and Infection, Department of Biology, Hull and York Medical School, University of York, York, United Kingdom
}

\section{A R T I C L E I N F O}

\section{Article history:}

Received 21 July 2013

Received in revised form 29 August 2013

Accepted 3 September 2013

\section{Keywords:}

HTLV-1

Proviral load

$\mathrm{HAM} / \mathrm{TSP}$

Cut-off value

Diagnosis

The geographic distribution of Human T-cell Lymphotropic Virus Type 1 (HTLV-1) infection makes one thing clear: except Japan, most of the estimated 20 million infected individuals are clustered within communities with limited health care access [1].

Given that the majority of infected persons remain disease-free, one of the challenges of the clinical management of HTLV-1infected patients with myelopathic symptoms is to establish the definite diagnosis of HTLV-Associated Myelopathy/Tropical Spastic Paraparesis (HAM/TSP). Asymptomatic carriers may complain of a range of symptoms that cannot be excluded from an association with HTLV-1, such as dry eyes, urinary incontinence and constipation. Current HAM/TSP diagnostic procedures are based

\footnotetext{
Abbreviations: HTLV, human T-cell lymphotropic virus; HAM/TSP, HTLV1-associated myelopathy/tropical spastic paraparesis; PBMC, peripheral blood mononuclear cells; PVL, proviral load.

* Corresponding author at: Advanced Laboratory of Public Health, Gonçalo Moniz Center, Fundacão Oswaldo Cruz - Bahia (FIOCRUZ), Rua Waldemar Falcão, 121, Candeal, Salvador, Bahia 40296-710, Brazil. Tel.: +55 7131762213; fax: +55 7131762000.

E-mail addresses: grassi@bahia.fiocruz.br, mfrgrassi@gmail.com (M.F.R. Grassi), vini_olavarria@hotmail.com (V.N. Olavarria),ramonkruschewsky@bahiana.edu.br (R.d.A. Kruschewsky), yyamano@marianna-u.ac.jp (Y. Yamano),

jacobsons@ninds.nih.gov (S. Jacobson),g.p.taylor@imperial.ac.uk (G.P. Taylor),

fabiola.martin@hyms.ac.uk (F. Martin), bgalvao@bahiana.edu.br (B. Galvão-Castro).
}

on criteria established by the World Health Organization (WHO), which consists of a list of neurological signs and symptoms in HTLV-1 seropositive subjects. In addition, imaging of the central nervous system is essential to exclude other neurological diseases with similar clinical features, whilst isolation of HTLV- 1 proviral in the cerebrospinal fluid (CSF) [2] is a positive finding, especially when the viral load in CSF lymphocytes is greater than in PBMCs [3]. However, these complementary tests are not readily available in many communities. In our experience, patients rarely present with all the essential features of HAM/TSP to meet the diagnostic requirements of the complete syndrome. To complement WHO criteria, a new classification strategy, the Belem Criteria, based on three diagnostic ascertainment levels was proposed [4]. HTLV-1infected patients with neurological defects are categorized as: (i) Definite HAM/TSP: patients who meet the established WHO criteria with a complete clinical presentation; (ii) Probable HAM/TSP: patients with a myelopathic mono-symptomatic presentation, in which other diseases resembling HAM/TSP have been excluded; (iii) Possible HAM/TSP: patients who present with a complete or incomplete clinical picture; however, other disorders resembling HAM/TSP cannot be excluded.

In recent years, several studies have demonstrated a clear association between HAM/TSP and HTLV-1 proviral load [5-11]. Moreover, compelling evidence indicates that patients with other 
Table 1

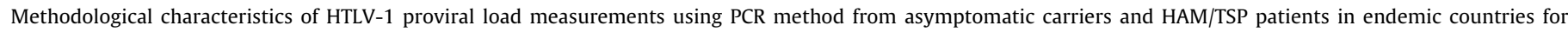
HTLV-1-infection.

\begin{tabular}{|c|c|c|c|c|c|c|c|}
\hline Author, year & Country & PCR method & Region amplified & $\begin{array}{l}\% \text { infected cells } \\
\text { asymptomatic }\end{array}$ & $\begin{array}{l}\text { Number of } \\
\text { asymptomatic carriers }\end{array}$ & $\begin{array}{l}\% \text { infected cells } \\
\text { HAM/TSP }\end{array}$ & $\begin{array}{l}\text { Number of } \\
\text { HAM/TSP patients }\end{array}$ \\
\hline Nagai et al., 1998 [8] & Japan & TaqMan & $\mathrm{pX}$ & $0.3^{\mathrm{a}} / 3.2^{\mathrm{a}}$ & 200 & 5.4 & 202 \\
\hline Manns et al., 1999 [7] & Jamaica & TaqMan & $\mathrm{pX}$ & 1.1 & 50 & 4.9 & 27 \\
\hline Olindo et al., 2005 [7] & Martinique & TaqMan & pol & 0.8 & 34 & 8.1 & 100 \\
\hline Montanheiro et al., 2005 [10] & Brazil & TaqMan & pol & 2.7 & 45 & 6.8 & 44 \\
\hline Best et al., 2006 [5] & Peru & SYBR green & $\mathrm{pX}$ & 5.6 & 33 & 18 & 35 \\
\hline Silva et al., 2007 [11] & Brazil & TaqMan & $\mathrm{pX}$ & 1.0 & 93 & 6.3 & 197 \\
\hline Grassi et al., 2011 [14] & Brazil & TaqMan & pol & 0.7 & 189 & 11.6 & 47 \\
\hline Furtado et al., 2012 [15] & Brazil & SYBR $^{\mathrm{a}}$ Green & pol & 0.5 & 75 & 3.4 & 78 \\
\hline Demontis et al., 2012 [3] & United Kingdom & SYBR Green & Tax & 1.8 & 211 & 14.7 & 85 \\
\hline
\end{tabular}

\#-Number of evaluated patients, \% infected cells: data represents median.

a DNA sample from whole blood. All other DNA samples were from peripheral blood mononuclear cells.

HTLV-1-associated inflammatory conditions, such as infective dermatitis [12] or keratoconjunctivitis sicca [13], display significantly higher levels of proviral load compared to asymptomatic carriers.

In a published study conducted in Bahia, Brazil, we found that a proviral load cut-off value of 50,000 copies $/ 10^{6}$ PBMCs (5\%), differentiated asymptomatic carriers from HAM/TSP patients with $87 \%$ sensitivity and $81 \%$ specificity [14]. Using the Belem criteria, only $22 \%$ of probable and $17 \%$ of definite HAM/TSP patients' HTLV-1 proviral loads fell below this cut-off value. If this threshold had been included as an additional criterion to diagnose HAM/TSP, $73 \%$ of patients from the probable group would be reclassified as definite.

Furtado et al. [15] also attempted to establish a proviral load cut-off value to distinguish asymptomatic carriers from HAM/TSP patients in Minas Gerais (Brazil). Using whole blood, a cut-off of 114 HTLV-1 copies $/ 10^{4}$ white blood cells (1.14\%), offered $78.2 \%$ sensitivity and $28 \%$ specificity in patients with HAM/TSP. This value is considerably lower than what was observed in Bahia. As members of the HAM/TSP Clinical Trial Study Group (HAM/TSP-CTSG) we performed a literature review of proviral loads reported in asymptomatic carriers and HAM/TSP patients (Table 1 ). The percentage of infected cells was approximately $6 \times$ higher in HAM/TSP patients (median 6.8\%, 5.4-18\%) than in asymptomatic carriers (median $1.1 \%, 0.3-5.6 \%$ ). Despite overlapping ranges, the trends are consistent across studies. However, study groups used diverse methods to measure proviral load, including different regions of the targeted HTLV-1 genome, the DNA sample source, e.g. whole blood cells or isolated PBMCs and cells quantified from patient samples. In personal consultation with HAM/TSP CTSG members we established that $7.04 \%$ and $10 \%$ of patients with definite HAM/TSP, living in Bahia, Brazil and Japan respectively, have a proviral load of $<1 \%$ in PBMCs. These patients have only mild disease. In contrast, all definite HAM/TSP patients from UK or USA had a proviral load of $>1 \%$ [3]. Based on the reviewed data and our own observations across four centres (Bahia/Japan/UK/USA) a single cut-off for asymptomatic versus HAM/TSP or for definite versus probable HAM/TSP cannot be recommended.

We therefore propose an international pilot study in an attempt to achieve consistent results in order to evaluate HTLV-1 proviral load in PBMCs of asymptomatic carriers as well as patients with probable, possible and definite HAM/TSP living in HTLV-1 endemic and non-endemic areas. By testing these patients at several chosen established laboratories blinded to samples' origins, we hope to take initial steps towards the standardization of proviral load quantification technique, with the ultimate goal of determining a relevant proviral load cutoff value to distinguish asymptomatic carriers from HAM/TSP patients. At the same time, we recognize that, due to variation from assay to assay, there are limitations with respect to viral load quantification [16,17]. In the future, the HTLV research community might propose the incorporation of a WHO international standard to aid in clinical applications by soliciting assistance from such groups as Standardization of Genome Amplification Techniques or National Institute for Biological Standards and Control.

\section{Funding}

Fundação de Amparo à Pesquisa da Bahia (FAPESB).

\section{Competing interests}

None declared.

\section{Ethical approval}

Not required.

\section{References}

[1] Hlela C, Shepperd S, Khumalo NP, Taylor GP. The prevalence of human T-cell lymphotropic virus type 1 in the general population is unknown. AIDS Rev 2009;11(4):205-14.

[2] WHO. Report on HTLV-1 Infection and Associated Diseases. Kagoshima: World Health Organization Scientific Group; 1989.

[3] Demontis MA, Hilburn S, Taylor GP, Human T. Cell lymphotropic virus type 1 viral load variability and long-term trends in asymptomatic carriers and in patients with human T cell lymphotropic virus type 1-related diseases. AIDS Res Hum Retroviruses 2013;29(2):359-64.

[4] De Castro-Costa CM, Araujo AQ, Barreto MM, et al. Proposal for diagnostic criteria of tropical spastic paraparesis/HTLV-I-associated myelopathy (TSP/HAM). AIDS Res Hum Retroviruses 2006;22(10):931-5.

[5] Best I, Adaui V, Verdonck K, et al. Proviral load and immune markers associated with human T-lymphotropic virus type 1 (HTLV-1)-associated myelopathy/tropical spastic paraparesis (HAM/TSP) in Peru. Clin Exp Immunol 2006;146(2):226-33.

[6] Lezin A, Olindo S, Oliere S, et al. Human T lymphotropic virus type I (HTLVI) proviral load in cerebrospinal fluid: a new criterion for the diagnosis of HTLV-I-associated myelopathy/tropical spastic paraparesis? J Infect Dis 2005;191(11):1830-4.

[7] Manns A, Miley WJ, Wilks RJ, et al. Quantitative proviral DNA and antibody levels in the natural history of HTLV-I infection. J Infect Dis 1999;180(5):1487-93.

[8] Nagai M, Usuku K, Matsumoto W, et al. Analysis of HTLV-I proviral load in 202 HAM/TSP patients and 243 asymptomatic HTLV-I carriers: high proviral load strongly predisposes to HAM/TSP. J Neurovirol 1998;4(6):586-93.

[9] Olindo S, Lezin A, Cabre P, et al. HTLV-1 proviral load in peripheral blood mononuclear cells quantified in 100 HAM/TSP patients: a marker of disease progression. J Neurol Sci 2005;237(1/2):53-9.

[10] Montanheiro P, Oliveira A, Posada-Vergara M, et al. Human T-cell lymphotropic virus type I (HTLV-I) proviral DNA viral load among asymptomatic patients and patients with HTLV-I-associated myelopathy/tropical spastic paraparesis. Braz J Med Biol Res 2005;38(11):1643-7.

[11] Silva MT, Harab RC, Leite AC, Schor D, Araujo A, Andrada-Serpa MJ. Human T lymphotropic virus type 1 (HTLV-1) proviral load in asymptomatic carriers, HTLV-1-associated myelopathy/tropical spastic paraparesis, and other neurological abnormalities associated with HTLV-1 infection. Clin Infect Dis 2007;44(5):689-92.

[12] Primo J, Siqueira I, Nascimento MC, et al. High HTLV-1 proviral load, a marker for HTLV-1 associated myelopathy/tropical spastic paraparesis, is also detected in patients with infective dermatitis associated with HTLV-1. Braz J Med Biol Res 2009;42(8):761-4. 
[13] Castro-Lima Vargens C, Grassi MF, Boa-Sorte N, et al. Keratoconjunctivitis sicca of human T cell lymphotropic virus type 1 (HTLV-1) infected individuals is associated with high levels of HTLV-1 proviral load. J Clin Virol 2011;52(3):177-80

[14] Grassi MF, Olavarria VN, Kruschewsky Rde A, et al. Human T cell lymphotropic virus type 1 (HTLV-1) proviral load of HTLV-associated myelopathy/tropical spastic paraparesis (HAM/TSP) patients according to new diagnostic criteria of HAM/TSP. J Med Virol 2011;83(7):1269-74.

[15] Furtado Mdos S, Andrade RG, Romanelli LC, et al. Monitoring the HTLV-1 proviral load in the peripheral blood of asymptomatic carriers and patients with HTLV-associated myelopathy/tropical spastic paraparesis from a Brazilian cohort: ROC curve analysis to establish the threshold for risk disease. J Med Virol 2012;84(4):664-71.

[16] Pang XL, Fox JD, Fenton JM, Miller GG, Caliendo AM, Preiksaitis JK. Interlaboratory comparison of cytomegalovirus viral load assays. Am J Transplant 2009;9(2):258-68.

[17] Preiksaitis JK, Pang XL, Fox JD, Fenton JM, Caliendo AM, Miller GG. Interlaboratory comparison of epstein-barr virus viral load assays. Am J Transplant 2009;9(2):269-79. 